\title{
APPLICATION OF SMART MATERIALS TO WIRELESS ID TAGS AND REMOTE SENSORS
}

\author{
RICHARD FLETCHER, JEREMY A. LEVITAN, JOEL ROSENBERG, \\ NEIL GERSHENFELD; The Media Laboratory, Massachusetts Institute of Technology, \\ Cambridge, MA 02139
}

\begin{abstract}
Material structures having an electromagnetic or magnetomechanical resonance can be excited or detected remotely using an antenna. Incorporating smart materials into such structures provides new opportunities to encode ID and sensor information in the electromagnetic signature of the "tag." In this way, it is possible to create tags which not only have a unique ID but which can also respond to local changes in their environment (e. g. force, temperature, light, etc.). This principle forms the basis for a low-cost wireless ID and wireless sensor technology which has many potential applications in manufacturing, inventory control, security, surveillance, and new human-computer interfaces. As a means of illustrating this concept, two simple examples are given: a force sensor incorporating a piezoelectric polymer and a relative position sensor which incorporates a magnetoelastic amorphous metal ribbon.
\end{abstract}

\section{INTRODUCTION}

When probed by an electromagnetic field, magnetoelastic amorphous metal ribbons and planar inductor-capacitor (LC) structures exhibit a discrete resonant frequency and Qfactor. Operating at kilohertz and megahertz frequencies, respectively, these resonators can be engineered to encode identification or information about the local environment. Common approaches to electromagnetic tagging include RFID (radio frequency identification) or some type of IC chip with a sensor. The disadvantages of these schemes, however, is their inability to meet the needs of applications where the total cost of each sensor must fall below $\$ 0.10$. The materials-based tagging technology described can meet this cost requirement and address a wide variety of applications, including disposable temperature probes, wireless force sensing devices, and small-scale remote identification.

Sensors based on LC resonators or magnetoelastic ribbons have been widely explored; however, most of these implementations are not wireless ${ }^{1}$ or are performed as an inductive magnetic measurement in very close proximity to the sensing material ${ }^{2}$. To enable operation over non-trivial distances ( $>$ few centimeters), the material structures presented here employ a resonant mode of operation. As wireless sensors, the the sensor and ID 
information is generally encoded in the resonant frequency and Q of the tag, which is then read from a distance ( 2 meters or less) using a near-field antenna [Fig 1]. Object identification can be accomplished, for example, by using multiple magnetoelastic strips or multiple-layer planar resonators to yield many unique identities. If the magnetoelastic ribbons is used in conjunction with a small permanent magnet, the bias-field dependence of the resonant frequency can be used as a mechanism for sensing relative displacement or force; and in planar LC resonators, incorporating a smart material dielectric would provide a means of sensing an external stimulus (e.g. force, temp, light). Given a properly designed sensor package, a pyroelectric dielectric leads to a temperature sensor and a piezoelectric dielectric provides a pressure sensor, for example.

This paper focuses on the use of such structures as sensor tags. Two types of sensors are illustrated in the sections that follow, and the corresponding data is given.
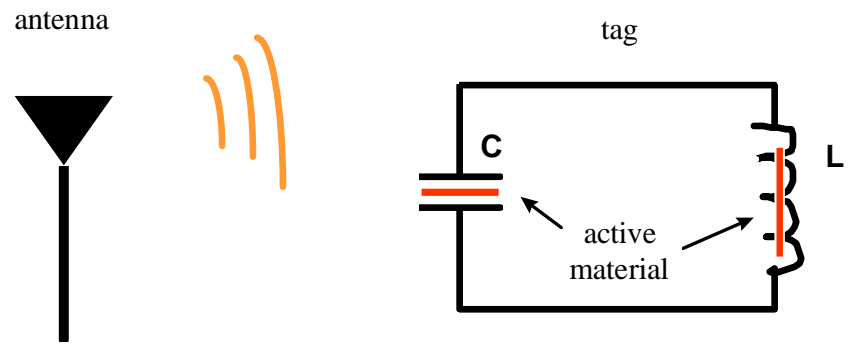

$$
\mathbf{w}_{0}=\left(L^{*} C\right)^{\wedge} .5, \quad 1 / Q \sim \sum \text { losses }
$$

Figure 1. Schematic representation of a materials-based wireless sensor tag exhibiting an electromagnetic or magnetomechanical resonance.

\section{SENSOR DESIGN AND EVALUATION}

A simple displacement sensor was evaluated for the purpose of measuring the linear position of a piston in a small cylinder (10 cm long). A strip of amorphous metal ribbon packaged in a plastic cavity was attached to the body of the cylinder. Additionally, a weak flat bias magnet made of Arnochrome $3^{\mathrm{TM}}$ of $\sim .5$ Oe or so can be included with the ribbon to provide a small constant bias field; however, for simplicity this aditional magnet was not used for this paper. The magnetoelastic ribbons used were amorphous alloys manufactured by Allied Signal and prepared in a width of $1.2 \mathrm{~cm}$ and length of $3.55 \mathrm{~cm}$. A properly oriented permanent magnet was attached to the end of the piston, thus providing a bias field to the ribbon which varied with linear position of the piston but did not vary with azimuthal rotation of the piston shaft [Figure 2]. 


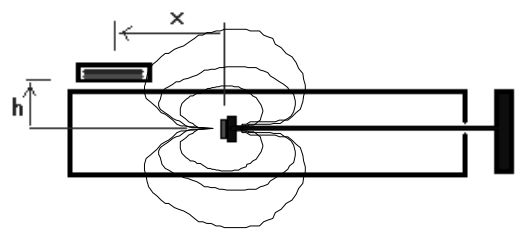

Figure 2. Schematic view of cylinder with position sensor.

Since the resonant behavior or the ribbon depends on the bias field as well as its material properties ${ }^{3}$, the linear position of the piston could then be deduced by tracking the resonant frequency of the ribbon. The dependence of the local bias magnetic field presented to tag as a function of piston position could be varied by changing the mounting position of the tag on the cylinder; and the resulting resonant frequency shift resulting from this field could be also be tuned independently through annealing treatments of the amorphous metal ribbon.

In order to increase the linear distance over which the sensor could operate, a preliminary annealing study was carried out to investigate the optimum processing parameters for the ribbons that were tailored to this application. Since it was desirable to increase the usable range of bias fields, a slightly sheared $\mathrm{M}-\mathrm{H}$ loop is desirable, so a transverse-field anneal was used. Samples of composition $\mathrm{Fe}_{38} \mathrm{Ni}_{39} \mathrm{Mo}_{2.4} \mathrm{~B}_{1} \mathrm{Si}_{0.2}$ were annealed at a temperature near 400 degrees Celsius using several different annealing fields. A second alloy used for this study was $\mathrm{Fe}_{35} \mathrm{Ni}_{33} \mathrm{Co}_{19} \mathrm{~B}_{8} \mathrm{Si}_{5}$, which was annealed by Sensormatic using another recipe. The resonant frequency shift as a function of an applied DC bias field was then measured using an Hewlett-Packard 8753D Network Analyzer. A representative sample of the measured data is shown in Figure 3. For $\mathrm{Fe}_{38} \mathrm{Ni}_{39} \mathrm{Mo}_{2.4} \mathrm{~B}_{1} \mathrm{Si}_{0.2}$, we suspect the annealing temperature was slightly higher than optimum, as exhibited by the extra degree of flattening in the curves likely due to partial recrystallization of the amorphous metal.
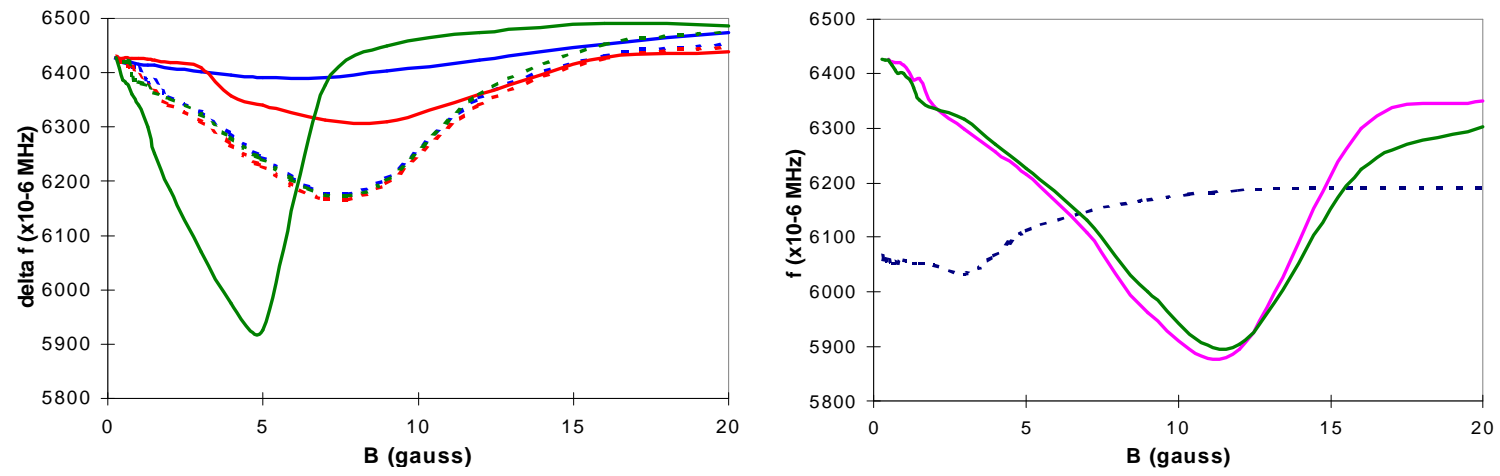

Figure 3. Bias-field dependence of the resonant frequency as a function of annealing treatment. On the left is data for $\mathrm{Fe}_{38} \mathrm{Ni}_{39} \mathrm{Mo}_{2.4} \mathrm{~B}_{1} \mathrm{Si}_{0.2}$ for transverse annealing fields of 0 , 200, 300 Oe. (curves for higher fields are flatter) On the right, is the result for $\mathrm{Fe}_{35} \mathrm{Ni}_{33} \mathrm{Co}_{19} \mathrm{~B}_{8} \mathrm{Si}_{5}$ after the Sensormatic annealing treatment. Dotted lines denote preannealed as-cast result. 


\section{Force Sensor Design \& Evaluation:}

For characterizing the planar tank's response to pressure, a two-coil planar resonator was designed and etched from copper clad 1000K120 Kapton made by Rogers. The two coils were then folded upon each other, with a $25 \mu \mathrm{m}$ dielectric placed between the two layers. The dielectric region was comprised of either teflon sheet or the piezoelectric polymer polyvinyldifluoride (PVDF), supplied by AMP, Inc. The structure was then epoxied under vacuum or laminated to seal the dielectric between the layers.

The performance of the sensor tag was evaluated using an Instron 4411 mechanical tester and the HP network analyzer. The sensor tag was placed over a 2" diameter sapphire base containing a loop antenna. The whole assembly was placed onto the lower anvil of an Instron machine. On top of the coil assembly, a 3" x 3" x 3" cube of nonconducting foam was placed between the sensor tag and the top anvil. Both the network analyzer and Instron press were connected to a PC via a GPIB interface. The Instron was programmed to apply a load in increments and output both the applied load and displacement from the origin. The resonant frequency and Q-factor for of the sensor tag was simultaneously recorded. These numbers form the basis for the data. The specific force sensor demonstrated in this paper is designed to have a sensing range similar to a human finger, so an applied load range of 0-5 Newtons was chosen.

\section{RESULTS AND DISCUSSION}

Position Sensor: One sample of each type of amorphous metal sample was selected for use as the sensor tag and mounted on the cylinder. The resonant frequency of each tag was then recorded as a function of the piston position. The results are shown in Figure 4.
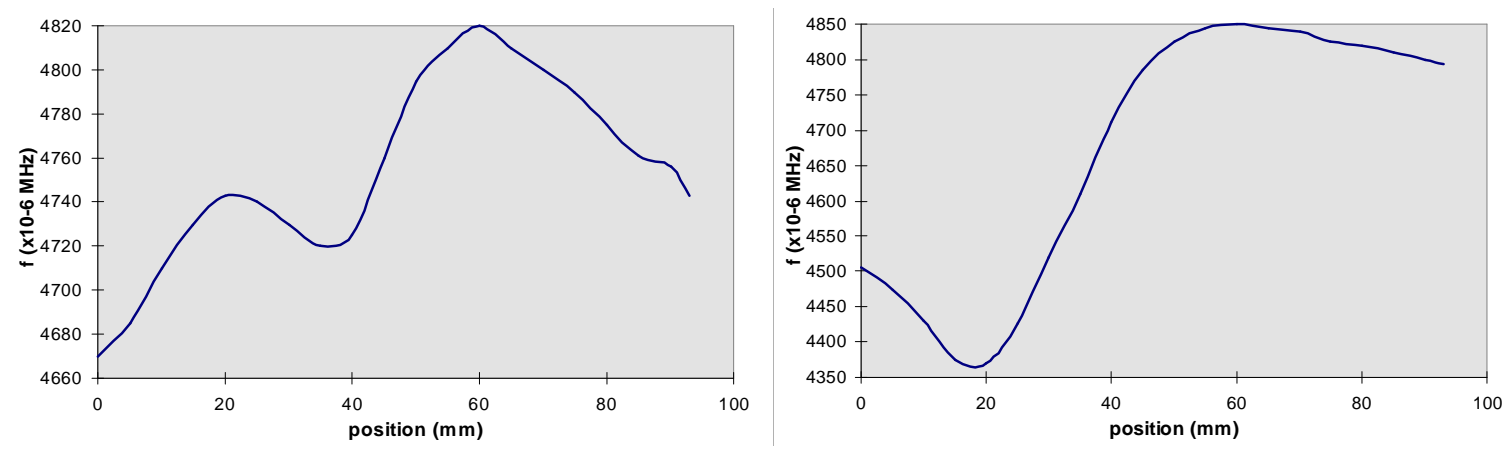

Figure 4. Plot of tag frequency vs. piston position for (left) and (right).

As shown in Figure 4, the usable sensing range was approximately $2 \mathrm{~cm}$ (from $\mathrm{x}=40$ $\mathrm{mm}$ to $\mathrm{x}=60 \mathrm{~mm}$ ) for $\mathrm{Fe}_{38} \mathrm{Ni}_{39} \mathrm{Mo}_{2.4} \mathrm{~B}_{1} \mathrm{Si}_{0.2}$, and was approximately $4 \mathrm{~cm}$ (from $\mathrm{x}=20$ mm to $\mathrm{x}=60 \mathrm{~mm}$ ) for $\mathrm{Fe}_{35} \mathrm{Ni}_{33} \mathrm{Co}_{19} \mathrm{~B}_{8} \mathrm{Si}_{5}$. By further optimizing the placement of the sensor tag as well as the strength of the permanent magnet used, we feel that the usable range of operation could be extended to $8 \mathrm{~cm}$ or more for a cylinder this size $(10 \mathrm{~cm}$ long). For a larger size cylinder, the design can be scaled up using a larger size 
magnetoelastic ribbon; or for very long cylinders, separate tags can be used along the length of the cylinder to track the piston position along the entire length of its stroke.

Force Sensor: The resonant frequency response of the planar tank circuits with PVDF dielectric was compared to that of tags containing Teflon, a conventional high-frequency dielectric. Results are plotted in Fig 5.

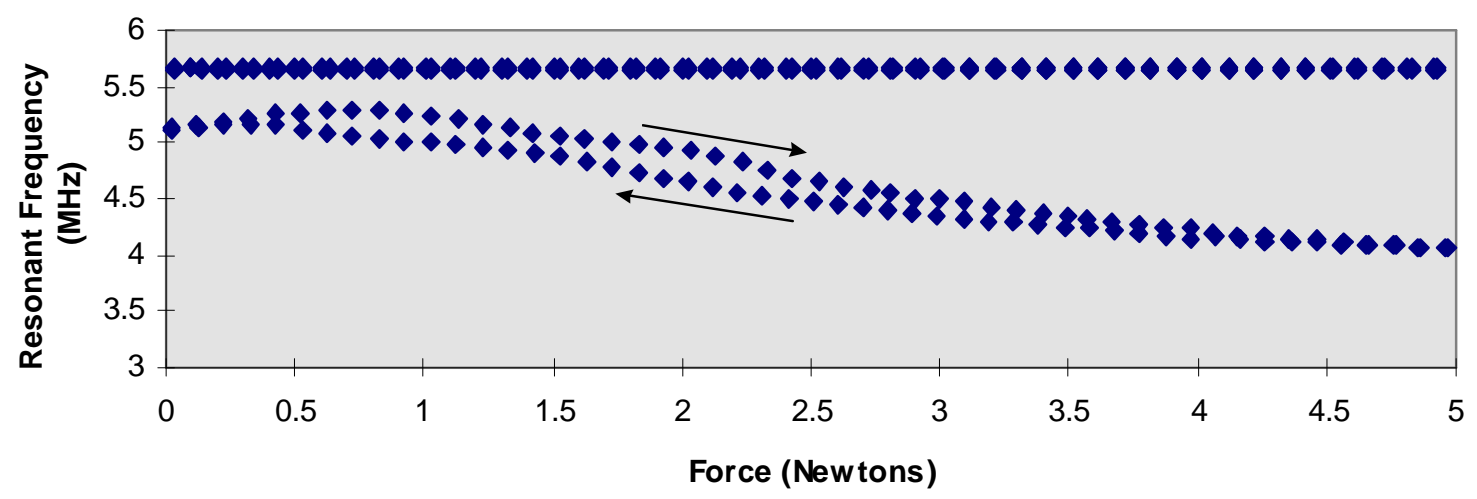

Figure 5. Data for wireless force sensor incorporating a piezoelectric polymer dielectric. The upper curve is the response of an identical resonant structure incorporating a normal dielectric material (teflon). Also plotted is the curve predicted by the linear elastic model.

For the resonator containing the normal dielectric, its response can be modeled as a simple LRC-circuit composed of an inductor, resistor, and plate capacitor with a dielectric material. By applying an elastic model to the deformation of the dielectric material under applied stress, the resonant frequency of the tag can be derived as a function of applied stress:

$$
\omega_{n}=\omega_{n_{0}} \cdot \sqrt{\frac{E-\sigma}{E}}
$$

where $\omega_{n_{0}}$ is the resonant frequency of the tag absent any applied stress, E is the Young's Modulus of the dielectric material, and $\sigma$ is the applied stress. Rearranging Equation (1) yields an expression relating the ratio of the change of resonant frequency versus initial resonant frequency and the induced strain, $\varepsilon$, in the dielectric material:

$$
\frac{\Delta \omega}{\omega_{n_{0}}}=1-\sqrt{1-\varepsilon}
$$

The curve predicted by this model is included in Figure 5 and very closely matched the measured data to within $0.1 \%$.

In comparing the teflon response to the response produced using PVDF, this model indicates that in a typical dielectric material with Young's Modulus of about $3 \mathrm{Gpa}$ (comparable to PVDF and clear teflon sheet), a 10\% change in frequency would occur when there is a strain of $19 \%$. Further manipulation of equation (2) shows that in order to produce in a $10 \%$ change in the resonant frequency of the tag, a force of 60000 Newtons 
would need to be applied to the tag. On the other hand, the smart material tag shows a significant response with an applied force of as little as 0.1 Newtons.

The primary advantages of the PVDF force sensor tag are its small thickness $(<0.5 \mathrm{~mm})$ and good sensitivity to small forces. The main apparent disadvantage of this sensor is hysteresis; however, we feel this can be partially attributed to the packaging of the PVDF and thus can be improved with better packaging design. This type of sensor has also proved to be quite robust, and continued to perform with no noticeable degradation in sensitivity even after subjecting the tag to abuse, such as stepping on it or striking it with a hammer. If the hysteresis is intrinsic to the PVDF, it seems more likely due to some type of repeatable relaxation mechanism exhibiting no noticeable degradation.

\section{SUMMARY}

Two simple examples of wireless "tag sensors" were illustrated which make use of smart materials. Such sensors can function as conventional sensor devices but are made of simple material structures and are wireless. As a result, these structures can represent a low-cost robust alternative to semiconductor-based sensor technology. Although further work is certainly required to quantitatively design, analyze and model the behavior of such tag sensors, this application has great potential for further research given the ongoing commercial interest in low-cost wireless sensor technology.

\section{ACKNOWLEDGEMENTS}

The authors would like to acknowledge Dr. Robert O'Handley of the MIT Material Science Department for helpful technical discussions, and Sensormatic Inc., AMP Inc., and Festo Inc. for donation of materials.

\section{REFERENCES}

${ }^{1}$ Mohri, IEEE Transactions on Magnetics, vol. MAG-20, No. 5, Sep 1984.

${ }^{2}$ For example: Seekircher and Hoffmann, Sensors and Actuators, A21-A23 (1990), pp. 401-405.

${ }^{3}$ Mermelstein, IEEE Transactions on Magnetics, vol. 28, no. 1, Jan 1992. 